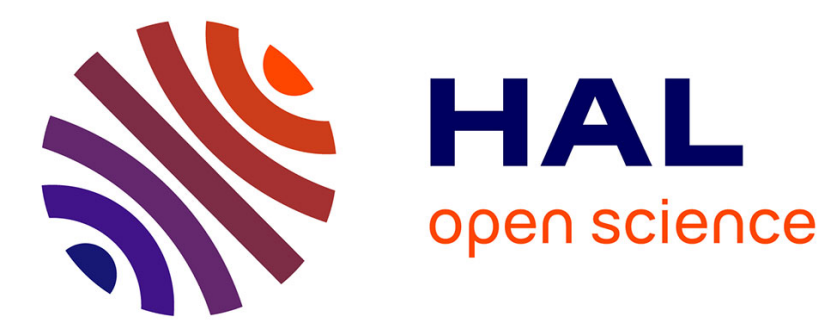

\title{
Quadratic Nonlinear Optics to Assess the Morphology of Riboflavin doped Chitosan for Eco-friendly Lithography
}

Cédric Ray, Mathieu Caillau, Christian Jonin, Emmanuel Benichou, Christophe Moulin, Estelle Salmon, Melissa E Maldonado, Anderson S L Gomes, Virginie Monnier, Emmanuelle Laurenceau, et al.

\section{To cite this version:}

Cédric Ray, Mathieu Caillau, Christian Jonin, Emmanuel Benichou, Christophe Moulin, et al.. Quadratic Nonlinear Optics to Assess the Morphology of Riboflavin doped Chitosan for Eco-friendly Lithography. Optical Materials, 2018, 10.1016/j.optmat.2018.04.007 . hal-01825534

\author{
HAL Id: hal-01825534 \\ https://hal.science/hal-01825534
}

Submitted on 28 Jun 2018

HAL is a multi-disciplinary open access archive for the deposit and dissemination of scientific research documents, whether they are published or not. The documents may come from teaching and research institutions in France or abroad, or from public or private research centers.
L'archive ouverte pluridisciplinaire HAL, est destinée au dépôt et à la diffusion de documents scientifiques de niveau recherche, publiés ou non, émanant des établissements d'enseignement et de recherche français ou étrangers, des laboratoires publics ou privés. 


\section{Quadratic Nonlinear Optics to Assess the Morphology of Riboflavin}

\section{doped Chitosan for Eco-friendly Lithography}

Cédric Ray ${ }^{1,}$, Mathieu Caillau $^{2}$, Christian Jonin ${ }^{1}$, Emmanuel Benichou $^{1}$, Christophe Moulin $^{1}$, Estelle Salmon ${ }^{1}$, Melissa E. Maldonado ${ }^{3}$, Anderson S.L. Gomes ${ }^{3}$, Virginie Monnier ${ }^{2}$, Emmanuelle Laurenceau ${ }^{2}$, Jean-Louis Leclercq ${ }^{2}$, Yann Chevolot ${ }^{2}$, Thierry Delair ${ }^{4}$ and PierreFrançois Brevet ${ }^{1}$

${ }^{1}$ Univ Lyon, Université Claude Bernard Lyon 1, UMR CNRS 5306, Institut Lumière Matière, F69622, Villeurbanne, France

${ }^{2}$ Univ Lyon, Ecole Centrale de Lyon, UMR CNRS 5270, Institut des Nanotechnologies de Lyon, F-69134, Ecully, France

${ }^{3}$ Departamento de Física, Universidade Federal de Pernambuco, 50670-901, Recife, PE, Brazil

4 Univ Lyon, Polytech, UMR-CNRS 5223, Ingénierie des Matériaux Polymères, F-69622, Villeurbanne

\section{Keywords}

Nonlinear optics, second harmonic generation, green lithography, riboflavin, chitosan

\section{Abstract}

We report the use of the Second Harmonic Generation response from a riboflavin doped chitosan film as a characterization method of the film morphology. This film is of particular interest in the development of new and bio-sourced material for eco-friendly UV lithography. The method allows us to determine how riboflavin is distributed as a function of film depth in the sample. This possibility is of importance in order to have a better understanding of the riboflavin influence in chitosan films during the lithography process. On the contrary, linear optical techniques provide no information beyond the mere confirmation of the riboflavin presence.

\footnotetext{
* Corresponding author cedric.ray@univ-lyon1.fr
} 


\section{Introduction}

Photolithography can nowadays achieve designs with high 2D spatial resolution for a wealth of industrial applications, notably in the field of nanoelectronics. This possibility results from the technical developments realized over the years in nanofabrication in the decrease in wavelength or the development and optimization of materials (masks, resists) and devices (lens, wavelength sources, exposure tools ...) for each technological node [1].

Photolithography is based on the use of photoresists generally composed of a polymer, a solvent, a photoactive component as a photo-acid generator, a quencher and different additives. This polymer solution is coated on the substrate leading to a thin film then patterned through photolithography. After development, it forms a temporary mask that protects selected areas of the underlying substrate allowing for the micro/nanopatterning (by etching or depositing matter locally) of the substrates for the fabrication of the devices. However, with a growing concern on environmental and health issues, new green photoresists are required. In this context, chitosan as a natural polymer, sub-product of the chitin shells of shrimps and other crustaceans, offers interesting possibilities. Its doping with riboflavin, a natural dye, seems promising.

Chitosan is a polysaccharide constituted of D-glucosamine and $\mathrm{N}$-acetyl-D-glucosamine units linked by a $\beta$ - $(1 \rightarrow 4)$ glycosidic bond (Figure 1$)$. This compound is naturally present in insects and microorganisms but can be also industrially prepared from chitin by treatment with an alkaline solution [2]. Chitin is the second most abundant biopolymer on Earth. It is extracted from wastes of seafood industry like crustacean shells from crabs and shrimps in particular. Chitosan is nontoxic, biocompatible and biodegradable At acidic $\mathrm{pH}$, chitosan is soluble in aqueous solutions. Riboflavin or vitamin $B_{2}$ (Figure 1) is a natural and hydrosoluble dye present in fruits, vegetables and animals [3]. It is known for its photosensitivity and used as a photodegradation agent of organic pollutants in water treatment [4]. Riboflavin-doped photosensitive chitosan films can be used for an eco-friendly and water-developable photolithography.

In photolithography, the polymer solution is spin-coated on a substrate prior to illumination. Therefore, novel characterization methods need to be proposed to determine the physical properties of the polymer thin film in view of the targeted eco-friendly lithography. In particular, the morphology of the film must be determined since the riboflavin distribution 
within the film is of utmost importance. Indeed, as the solvent is evaporated from the film, the solubility limit of riboflavin (around $2 \times 10^{-4} \mathrm{~mol}^{-1} \mathrm{~L}^{-1}$ ) may be reached leading to phase segregation whereas a homogeneous distribution is desired. Linear optical methods like UVvisible absorption and fluorescence spectroscopies are among the simplest methods and indeed provide valuable information. Chitosan, although presenting a weak fluorescence, has no prominent features in the visible part of the spectrum as opposed to riboflavin which possesses both strong absorption and fluorescence bands. Yet, linear optical methods are known to lack longitudinal resolution along the direction of propagation and are therefore of limited use when dopant depth profiles are sought at micrometer resolution or better.

In order to improve the longitudinal resolution of the optical characterization, we propose in this work to use different complementary nonlinear optical methods. First, we have applied the two photon excited fluorescence (TPEF) method which permits to obtain a threedimensional resolution owing to the multiphoton excitation process [5-7]. Indeed, because two photons are simultaneously required to achieve material excitation, the nonlinear optical process can only occur at the focus of the beam [8]. Thus, with a typical focal volume of $10 \mu \mathrm{m}^{3}$, a depth resolution of few micrometers in the plane perpendicular to the propagation direction can be achieved. However, the linear and nonlinear methods have a similar resolution limited by diffraction and are not so well suited to investigate the film interfaces. Therefore, we propose to also perform Second Harmonic Generation (SHG) measurements at the air-film interface [9]. The SHG process whereby two photons at the fundamental frequency are converted into one photon at the harmonic frequency, is highly sensitive to the symmetry of the material and in particular, is forbidden in media with inversion symmetry within the dipole approximation, that is within the volume of the film. Hence, the method is inherently surface sensitive and should allow us to go further in assessing any presence or accumulation of riboflavin at the air-film interface. The surface sensitivity is here determined by the extent of the centrosymmetry breaking into the film. The interface is a region where the centrosymmetry is broken whereas the volume is centrosymmetric by nature. Similarly to liquids, this interface region where the centrosymmetry is broken should not exceed a few molecular layers, i.e. should be of the order of a nanometer. With the combination of TPEF and SHS, to determine the riboflavin distribution in the film volume, and SHG, to determine its presence or absence at the film interfaces, one can thus retrieve a full picture of the 
riboflavin spatial distribution. In fact, after the first few atomic or molecular layers, any centrosymmetric media will exhibit no coherent SHG signal. Only the symmetry breaking due a media change at the surface, can give any signal for a centrosymmetric media.

From the molecular structure of riboflavin which contains delocalized electrons in an asymmetric environment (Figure 1), we can expect that the molecule exhibits a significant first hyperpolarizability, namely its cross-section for SHG, and thus a significant second harmonic intensity. The first hyperpolarizability of a molecular compound can be determined by the Second Harmonic Scattering (SHS) method [10-12], also referred as incoherent SHG. In this case, the harmonic light results from the scattering by a molecular suspension in a liquid due to orientation fluctuations. In this paper, we therefore report the SHG response from a riboflavin doped chitosan film deposited on a silicon substrate supporting a $200 \mathrm{~nm}$ silica layer. We first determined the film linear optical properties and the riboflavin first hyperpolarizability when dispersed in bulk solution using the SHS technique. Then, the TPEF and SHG intensities of riboflavin in thin chitosan films were recorded. The 2D spatial distribution of the molecules in the film was characterized by nonlinear imaging. Finally, surface SHG measurements were performed in order to confirm the absence of riboflavin at the air-film interface.

\section{Experimental Section}

\section{Chemistry}

Riboflavin was purchased from Sigma-Aldrich (> $98 \%$ ). Chitosan was purchased from Mahtani Chitosan PVT, Ltd (India) and was characterized by its molecular weight and its degree of acetylation (DA) corresponding to the number of $\mathrm{N}$-acetyl-D-glucosamine units along a chain of chitosan. In our case, chitosan was extracted from squid pens with a DA of $2 \%$ and a molar mass of $569.9 \mathrm{~kg} \cdot \mathrm{mol}^{-1}$.

A riboflavin solution concentrated at $2.2 \times 10^{-4} \mathrm{~mol}^{\mathrm{L}} \mathrm{L}^{-1}$ in deionized water (ultra-pure, 18 $\mathrm{M} \Omega . \mathrm{cm}$ ) was prepared. Chitosan was dissolved in this solution at a concentration of $0.9 \%$ (w/v). To enable its total dissolution, acetic acid (Fluka Analytical, glacial acetic acid) was added at a final concentration of 0.05 mol. $\mathrm{L}^{-1}$. Riboflavin doped chitosan films were realized by spin-coating (5000 rd.min-1, $3000 \mathrm{rd} . \mathrm{s}^{-2}$ during $30 \mathrm{~s}$ ) on n-doped (100) oriented Si substrates with a $200 \mathrm{~nm}$ thick $\mathrm{SiO}_{2}$ layer deposited by plasma enhanced chemical vapor 
deposition (PECVD). After spin-coating, the resulting film was heated at $100{ }^{\circ} \mathrm{C}$ for $1 \mathrm{~min}$. The riboflavin-doped chitosan film thickness was $180 \mathrm{~nm} \pm 5 \mathrm{~nm}$. Film thickness was measured by ellipsometry with a spectroscopic ellipsometer UVISEL ${ }^{\mathrm{TM}}$ from Horiba using the classical formula dispersion as modelled in the Horiba software. The model was adapted to chitosan by adjusting the refractive index between 1.5-1.6 and the extinction coefficient to 0 at 630 $\mathrm{nm}$ following Nosal et al. [13].

\section{Exposure}

Films were exposed to UV radiations through a photomask constituted of open zones (lines of $60 \mu \mathrm{m}$ ) in quartz and dark zones (squares of $440 \mu \mathrm{m} \times 440 \mu \mathrm{m}$ ) in chromium-iron oxide. Exposure was realized during 1 minute on a MBJB4 UV SUSS Micro Tec UV lamp at $365 \mathrm{~nm}$, $405 \mathrm{~nm}$ and $435 \mathrm{~nm}$ with a power density of $20 \mathrm{~mW} \cdot \mathrm{cm}^{-2}$ at $405 \mathrm{~nm}$ and a lamp power of 200 W. Patterns were revealed in the riboflavin-doped chitosan films after development in deionized water for $60 \mathrm{~s}$. Non exposed films were stored in clean room in the dark after preparation [14].

\section{Linear Optics}

Ultraviolet-Visible absorption spectra were collected in a quartz cell with $1 \mathrm{~cm}$ path length using a double beam UV mc2 SAFAS spectrometer. One-photon excited fluorescence spectra were performed with a FLS920 fluorimeter from Edinburgh Photonics using quartz cells with 1 $\mathrm{cm}$ path length. A $450 \mathrm{~W}$ continuous xenon arc lamp was used as an excitation source.

\section{Nonlinear optics}

The nonlinear imaging experimental setup was based on a frequency doubled femtosecond Er-doped fiber laser source (Menlo Lasers, C-Fiber 780). The laser provided pulses with a duration of about $100 \mathrm{fs}$ at a repetition rate of $100 \mathrm{MHz}$. The fundamental wavelength was set at $780 \mathrm{~nm}$ (spectral width of $13 \mathrm{~nm}$ ) and an average power of about $65 \mathrm{~mW}$ was measured at the laser exit. The light beam was focused by a standard microscope objective (magnification X20, NA 0.75) mounted onto a microscope stand (Nikon Eclipse TE2000-U with 3D positioning in inverted geometry) holding the sample. Galvanometric mirrors allowed for the beam to be swept to acquire non-linear images of various samples. The nonlinear optical signals, SHG and TPEF, were collected into an optical fiber feeding a spectrometer after going through various color filters. These filters were placed on the emission light beam path to 
avoid any unwanted fundamental or SHG light and also to separate TPEF and SHG signals. A cooled photomultiplier tube was placed after the spectrometer.

Hyperpolarisability measurements were performed in a SHS setup as described in Duboisset et al [15]. Briefly, incoherent second harmonic emission is generated in a solution illuminated by a femtosecond laser focused by a microscope objective and measured, after collection by a microscope objective, on a photomultiplier installed on a spectrometer.

The detailed description of the SHG setup can be found elsewhere [16]. Briefly, in our configuration, a fundamental $780 \mathrm{~nm}$ incoming wave was impinging onto the film surface with a $70^{\circ}$ angle with controlled linear polarization. The harmonic outgoing wave at $390 \mathrm{~nm}$ was then collected in specular reflection through a spectrometer and a CCD camera [16].

\section{Results and Discussion}

\section{Riboflavin properties in solution}

In order to investigate the properties of Riboflavin-doped Chitosan films we first discuss the optical properties of the Chitosan/Riboflavin solutions used to produce the films. Within the UV-visible spectral range, the optical features in absorption and fluorescence are essentially provided by riboflavin with a secondary contribution from chitosan. The UV-visible absorption spectrum of the initial riboflavin solution used in the film fabrication is shown in Figure 2. The absorbance of the $0.9 \%$ chitosan solution is negligible in the visible spectrum and is only present in the UV range. On the opposite, the $2.2 \times 10^{-4} \mathrm{~mol}^{-1} \mathrm{~L}^{-1}$ riboflavin solution exhibits four peaks, two in the visible and two in the UV range albeit at higher wavelengths as compared to chitosan.

Because the lithography process entails a UV light exposure of the film, a comparison is made with an exposed riboflavin + chitosan solution. Exposure was performed at $365 \mathrm{~nm}, 405 \mathrm{~nm}$ and $435 \mathrm{~nm}$. The absorption spectrum of the exposed solution differs quite dramatically from the non-exposed riboflavin. In fact, its absorption is very similar to that of lumichrome, the reaction product of the riboflavin photodegradation [17].

As shown in Figure 3, fluorescence spectra were also recorded under single photon excitation at $377 \mathrm{~nm}$. Chitosan is known to present fluorescence [19]. However, in our experiments, its fluorescence was very weak. The observed fluorescence spectrum therefore closely matches that of the Riboflavin and the mixture solutions. Since our chitosan solution yields a much 
lower fluorescence signal [18] compared to riboflavin solution, the riboflavin and chitosan mixture solution closely matches the riboflavin spectrum.

We estimated also the first hyperpolarizability of riboflavin. The SHG scattered intensity was collected in a right angle geometry for different dilutions of a riboflavin aqueous solution. When normalized to the intensity of the neat solvent these samples exhibit a linear behavior and the slope $p$ is directly given by:

$$
p=\frac{\left\langle\beta^{2}\right\rangle}{N_{w}\left\langle\beta_{w}^{2}\right\rangle}
$$

where $N_{w}=55.56 \mathrm{M}$ is the number of water molecules per unit volume and $\left\langle\beta_{w}^{2}\right\rangle$ and $\left\langle\beta^{2}\right\rangle$ are the square of the first hyperpolarizability, for water and riboflavin respectively, in the laboratory frame averaged over all space orientation in accordance with the liquid phase isotropy. The measurement was conducted at room temperature with a fundamental wavelength of $780 \mathrm{~nm}$ leading to the estimation of a value of $\sqrt{\left\langle\beta^{2}\right\rangle}=(2.5 \pm 0.5) \times 10^{-28}$ esu using the $\left\langle\beta_{w}^{2}\right\rangle$ water reference given by Duboisset et al. [15]. This value, as expected, is significant if compared to 4-(4-dihexadecylaminostyryl)-N-methylpyridinium iodide, a compound well known for its large hyperpolarizability of $(17.6 \pm 0.5) \times 10^{-28}$ esu [19]. Thus, the detection of riboflavin with SHG was readily accessible although the experimental conditions were not optimal.

\section{Riboflavin-chitosan film properties}

First, the reflectance properties of the film were determined. Figure 4 shows that the film linear optical properties derive from chitosan only since the riboflavin content is too low.

Note that in Figure 4, the reflectance spectra clearly exhibit the interference fringes arising from the thin film.

Then, the nonlinear optical properties of the film were studied with TPEF. Figure 5 shows the TPEF spectra recorded for a pure chitosan film as well as a mixed riboflavin-chitosan film. Two photons, with a wavelength of $780 \mathrm{~nm}$, were used and absorbed. This excitation can be compared to an irradiation at $390 \mathrm{~nm}$ (half the initial wavelength) which is close to the excitation used to acquire the one photon fluorescence spectra $(377 \mathrm{~nm})$. The fluorescence maximum was measured at a wavelength of about $500 \mathrm{~nm}$ with a shoulder at shorter wavelengths, around $450 \mathrm{~nm}$. 
On the opposite, the pure chitosan film showed almost no two-photon excited fluorescence signal. Interestingly, both films presented a SHG response located at half the $780 \mathrm{~nm}$ excitation wavelength. However, the SHG intensity seemed unaffected by the riboflavin presence and seems to therefore arise mainly from the pure chitosan film on the $\mathrm{SiO}_{2}$ substrate.

In order to characterize the distribution of riboflavin within the chitosan film, depth profiles were recorded for different wavelengths on the 2D pattern imprinted on the riboflavin doped chitosan film. Two distinct areas of the sample were selected to analyze the exposed and non-exposed regions, respectively identified with a red or a blue square on Figure 6 (right panel).

It is clear from Figure 6 that the non-exposed region provided less fluorescence than the exposed one.

In Figure 6, the neat $\mathrm{SiO}_{2}$ substrate, designated as 'reference' and shown in black, is obtained in absence of riboflavin. The shifted position is due to the uncertainty when positioning different samples. This depth profile measurement resulted from the convolution of the beam focus and the film depth. Hence a TPEF maximum indicated that at the resolution imposed by the beam waist (few micrometers) riboflavin is distributed rather homogeneously within the film.

Figure 7 provides the depth profile recorded for SHS at the same areas as in Figure 6 . The results are very similar to those obtained with TPEF and confirm that riboflavin was rather homogeneously distributed in the film. However, the larger contrast between the exposed and non-exposed areas obtained with SHG suggests that this method provides better contrasted images of the sample after UV exposure. The presence of riboflavin clusters with a size of tens of microns is also visible on the right panel of figure 7 (bright white signature). This feature probably arises for undissolved riboflavin crystals although riboflavin reaches its maximum of solubility under the conditions used. The solution was however not filtered.

Despite the rather homogeneous distribution of riboflavin molecules in the bulk of the film, it was impossible to ensure the absence of riboflavin accumulation at the surface of the film (first few molecular layers) with the profiles obtained. This feature can be accessed with surface SHG known to provide a molecular information on the first few molecular layers of 
centrosymmetric films like chitosan [20-22]. We emphasize here that for a centrosymmetric film, similarly to liquids, it is assumed that this breaking of centrosymmetry pertains over a few molecular diameters only, i.e. over distances of the order of $1 \mathrm{~nm}$. Any breaking over much larger distances, like micrometers, would be observed with the volume profile. Indeed, the SHG signal is only coming from the few molecular layers where the surface is not centrosymmetric. Moreover, the laser interaction with the bulk of the centrosymmetric film does not give any SHG signal.

We performed a polarization analysis of the surface SHG intensity collected from the air-film interface in presence and absence of riboflavin where the input fundamental beam angle of polarization was rotated for two perpendicular angles of the analyzer, respectively $\mathrm{S}$ and $\mathrm{P}$. Hence, two plots were recorded, the SHG intensities so-called IP and Is, for chitosan films and chitosan/riboflavin films under non-exposed conditions (Figure 8). Data obtained for other film compositions show very similar trends and are not shown. The plots all exhibit a two-lobe pattern for the P-polarized graph (squares) and a four-lobe pattern for the S- polarized graph (circles) of the SH intensity as a function of the input polarization angle, as expected from theory. These plots were analyzed using the standard simplified form of the SHG intensity as a function of the input polarization angle $\gamma$ [23]:

$$
\begin{aligned}
& I_{P} \propto\left|a \cdot \cos ^{2}(\gamma)+b \cdot \sin ^{2}(\gamma)\right|^{2} \\
& I_{S} \propto|c \cdot \sin (2 \gamma)|^{2}
\end{aligned}
$$

where $a, b$ and $c$ are three parameters depending on the geometrical configuration, the optical indices at 800 and $400 \mathrm{~nm}$ and the three non-vanishing independent tensor elements of the isotropic air-film interface, namely $\chi_{S, X X Z}^{(2)}, \chi_{S, Z X X}^{(2)}$ and $\chi_{S, Z Z Z}^{(2)}$.

\begin{tabular}{l|ccc}
\hline Si/SiO 2 substrate & $a$ & $a / c$ & $b / c$ \\
\hline Substrate & $183.6 \pm 0.6$ & $2.05 \pm 0.01$ & $-2.03 \pm 0.01$ \\
Substrate + Chitosan & $224.6 \pm 0.3$ & $5.81 \pm 0.05$ & $-1.14 \pm 0.03$ \\
Substrate + Chitosan + Riboflavin & $212.9 \pm 0.2$ & $5.90 \pm 0.05$ & $-1.18 \pm 0.02$ \\
Substrate + Chitosan + Riboflavin (insolated) & $221.1 \pm 0.4$ & $5.62 \pm 0.04$ & $-1.09 \pm 0.04$
\end{tabular}

Table 1 : Adjustment parameters and ratios normalized to the c coefficient, for the polarization resolved SHG intensity plots obtained using Eqs. (2a), (2b) for films on $\mathrm{Si}_{\text {SiO }}$ substrate. 
The adjustment procedure for all different films composition led to the numerical values reported in Table 1. The recorded SHG intensity was dominated by the $I_{P}$ component whereas the $I_{S}(\gamma)$-component was much weaker as shown on Figure 8. These results were identical for samples after and before exposition as well as for with pure chitosan films (left of Figure 8). Once the parameters are renormalized with respect to $c$, it is obvious that the parameters $a$ and $b$ are rather constant indicating that riboflavin does not accumulate at the surface. These results clearly demonstrate the absence of riboflavin in the first few molecular layers of the sample.

It is also clear that one observes a large increase of the $a$ coefficient upon the chitosan deposition on the surface of the neat substrate, as expected. Indeed the a coefficient is proportional to $I_{P}$, that is the $\mathrm{P}$ polarized intensity at both fundamental and harmonic frequency. This large increase is the signature of drastic changes at the surface when chitosan is deposited on the $\mathrm{Si} / \mathrm{SiO}_{2}$ bare substrate. This polarization configuration is the most

\begin{tabular}{l|ccc}
\hline \multicolumn{1}{c}{ Glass substrate } & $a$ & $a / c$ & $b / c$ \\
\hline Substrate & $46.98 \pm 0.05$ & $2.42 \pm 0.01$ & $0.318 \pm 0.007$ \\
Substrate + Chitosan & $64.80 \pm 0.07$ & $1.90 \pm 0.01$ & $0.428 \pm 0.005$ \\
Substrate + Chitosan + Riboflavin & $60.35 \pm 0.08$ & $2.05 \pm 0.01$ & $0.411 \pm 0.007$ \\
Substrate + Chitosan + Riboflavin (insolated) & $63.55 \pm 0.07$ & $2.02 \pm 0.01$ & $0.489 \pm 0.005$ \\
\hline
\end{tabular}

sensitive one to these changes.

Table 2 : Adjustment parameters and ratios normalized to the c coefficient, for the polarization resolved SHG intensity plots obtained using Eqs. (2a), (2b) for films on glass substrate.

In order to control further that the presence of riboflavin at the surface is independent of the substrate, we also analyzed the same films deposited on a glass substrate. The fit parameters of the adjustments are presented in 


\begin{tabular}{l|ccc}
\hline Glass substrate & $a$ & $a / c$ & $b / c$ \\
\hline Substrate & $46.98 \pm 0.05$ & $2.42 \pm 0.01$ & $0.318 \pm 0.007$ \\
Substrate + Chitosan & $64.80 \pm 0.07$ & $1.90 \pm 0.01$ & $0.428 \pm 0.005$ \\
Substrate + Chitosan + Riboflavin & $60.35 \pm 0.08$ & $2.05 \pm 0.01$ & $0.411 \pm 0.007$ \\
Substrate + Chitosan + Riboflavin (insolated) & $63.55 \pm 0.07$ & $2.02 \pm 0.01$ & $0.489 \pm 0.005$ \\
\hline
\end{tabular}

Table 2. As for the $\mathrm{Si} / \mathrm{SiO}_{2}$ substrate, the a coefficient exhibits an increase upon the deposition of a chitosan film, though the relative increase is much smaller. After normalization by the $c$ coefficient, one can note that the results are similar to the $\mathrm{Si} / \mathrm{SiO}_{2}$ substrate indicating no clear sign of riboflavin accumulation within the first few layers of the film interface. These results also illustrate the great sensitivity of surface SHG [23] to probe these film interfaces.

\section{Conclusion and perspectives}

We have reported the use of the nonlinear optical techniques of TPEF and SHG to investigate the distribution of riboflavin in a chitosan thin film deposited on a substrate. The results show that riboflavin does not accumulate at the air-film interface. Moreover, the SHG technique demonstrate a better characterization of the chitosan/riboflavin film interface than the TPEF technique. The nonlinear optical techniques used in this study are therefore highly complementary and efficient in determining the riboflavin depth distribution of the doped chitosan films. They should thus help improving eco-friendly lithography processes (especially UV exposure photo-mechanism in presence of dyes) by a better polymer film characterization.

As we have mentioned, some samples contained riboflavin clusters. Thus it would be interesting to decrease the riboflavin concentration in the chitosan solutions to avoid precipitation. The first lead would be to precisely determine the riboflavin solubility in chitosan in order to decrease its concentration while preserving the lithographic properties. Another important parameter to investigate is the degree of acetylation (DA) of the chitosan. Indeed, we used low DA (2\%) chitosan but higher DA ones (up to 50\%) may affect the riboflavin film solubilization through a synergic interaction between the acetamide group and riboflavin. 


\section{Acknowledgements}

This work is supported by the LABEX iMUST (ANR-10-LABX-0064) of Universite de Lyon within the program "Investissements d'Avenir" (ANR-11-IDEX-0007) operated by the French National Research Agency (ANR). This work is performed thanks to the Centre of Nano-Optics Nanoptec of the University Claude Bernard Lyon 1 and the France-Brazil CAPES-COFECUB (Sv852-15) program is also acknowledged.

Nanolyon platform is acknowledged for equipment and support. Green lithography project is supported by Pulsalys. MC also acknowledges the EEA doctoral school for a doctoral scholarship.

\section{References}

1. K. Ronse, "Optical lithography-a historical perspective," Comptes Rendus Phys. 7, 844-857 (2006).

2. M. Rinaudo, "Chitin and chitosan: Properties and applications," Prog. Polym. Sci. 31, 603-632 (2006).

3. M. A. Sheraz, S. H. Kazi, S. Ahmed, Z. Anwar, and I. Ahmad, "Photo, thermal and chemical degradation of riboflavin," Beilstein J. Org. Chem. 10, 1999-2012 (2014).

4. J. P. Escalada, A. Pajares, J. Gianotti, A. Biasutti, S. Criado, P. Molina, W. Massad, F. Amat-Guerri, and N. A. García, "Photosensitized degradation in water of the phenolic pesticides bromoxynil and dichlorophen in the presence of riboflavin, as a model of their natural photodecomposition in the environment," J. Hazard. Mater. 186, 466-472 (2011).

5. P. T. C. So, C. Y. Dong, B. R. Masters, and K. M. Berland, "Two-Photon Excitation Fluorescence Microscopy," Annu. Rev. Biomed. Eng. 2, 399-429 (2000).

6. W. Denk, J. H. Strickler, and W. W. Webb, "Two-photon laser scanning fluorescence microscopy," Science 248, 73-76 (1990).

7. C. Xu and W. W. Webb, "Measurement of two-photon excitation cross sections of molecular fluorophores with data from 690 to 1050 nm," JOSA B 13, 481-491 (1996).

8. W. Kaiser and C. G. B. Garrett, "Two-Photon Excitation in CaF2:Eu2+," Phys. Rev. Lett. 7, 229-231 (1961).

9. Y. R. Shen, "Surface properties probed by second-harmonic and sum-frequency generation," Nature 337, 519-525 (1989).

10. K. Clays and A. Persoons, "Hyper-Rayleigh scattering in solution," Phys. Rev. Lett. 66, 2980-2983 (1991).

11. J. I. Dadap, J. Shan, K. B. Eisenthal, and T. F. Heinz, "Second-Harmonic Rayleigh Scattering from a Sphere of Centrosymmetric Material," Phys. Rev. Lett. 83, 4045-4048 (1999).

12. G. Revillod, I. Russier-Antoine, E. Benichou, C. Jonin, and P. Brevet, "Hyper Rayleigh Scattering of Molecular Aggregates," Nonlinear Opt. Quantum Opt. 35, 135 (2006). 
13. W. H. Nosal, D. W. Thompson, L. Yan, S. Sarkar, A. Subramanian, and J. A. Woollam, "UV-vis-infrared optical and AFM study of spin-cast chitosan films," Colloids Surf. B Biointerfaces 43, 131-137 (2005).

14. M. Caillau, C. Chevalier, P. Crémillieu, T. Delair, O. Soppera, B. Leuschel, C. Ray, C. Moulin, C. Jonin, E. Benichou, P.-F. Brevet, C. Yeromonahos, E. Laurenceau, Y. Chevolot, and J.-L. Leclercq, "Sub-micron lines patterning into silica using water developable chitosan bioresist films for eco-friendly positive tone e-beam and UV lithography," in Optical Microlithography XXXI (International Society for Optics and Photonics, 2018), Vol. 10587, p. 105870S.

15. J. Duboisset, A. Deniset-Besseau, E. Benichou, I. Russier-Antoine, N. Lascoux, C. Jonin, F. Hache, M.-C. Schanne-Klein, and P.-F. Brevet, "A bottom-up approach to build the hyperpolarizability of peptides and proteins from their amino acids," J. Phys. Chem. B 117, 9877-9881 (2013).

16. G. Martin-Gassin, E. Benichou, G. Bachelier, I. Russier-Antoine, C. Jonin, and P. F. Brevet, "Compression Induced Chirality in Dense Molecular Films at the Air-Water Interface Probed by Second Harmonic Generation," J. Phys. Chem. C 112, 12958-12965 (2008).

17. J. Koziol, "Absorption spectra of riboflavin, lumiflavin, and lumichrome in organic solvents," Experientia 21, 189-190 (1965).

18. H. Huang, F. Liu, S. Chen, Q. Zhao, B. Liao, Y. Long, Y. Zeng, and X. Xia, "Enhanced fluorescence of chitosan based on size change of micelles and application to directly selective detecting Fe3+ in humanserum," Biosens. Bioelectron. 42, 539-544 (2013).

19. G. Revillod, J. Duboisset, I. Russier-Antoine, E. Benichou, G. Bachelier, C. Jonin, and P.-F. Brevet, "Multipolar Contributions to the Second Harmonic Response from Mixed DiA-SDS Molecular Aggregates," J. Phys. Chem. C 112, 2716-2723 (2008).

20. Y. R. Shen, "Optical Second Harmonic Generation at Interfaces," Annu. Rev. Phys. Chem. 40, 327-350 (1989).

21. K. B. Eisenthal, "Liquid Interfaces Probed by Second-Harmonic and Sum-Frequency Spectroscopy," Chem. Rev. 96, 1343-1360 (1996).

22. A. G. Volkov and D. W. Deamer, Liquid-Liquid Interfaces Theory and Methods (CRC Press, 1996).

23. A. Bruyère, E. Benichou, L. Guy, A. Bensalah-Ledoux, S. Guy, and P.-F. Brevet, "Reversibility of the supramolecular chirality of bridged binaphtol derivatives at the airwater interface," Opt. Mater. Express 4, 2516-2524 (2014). 


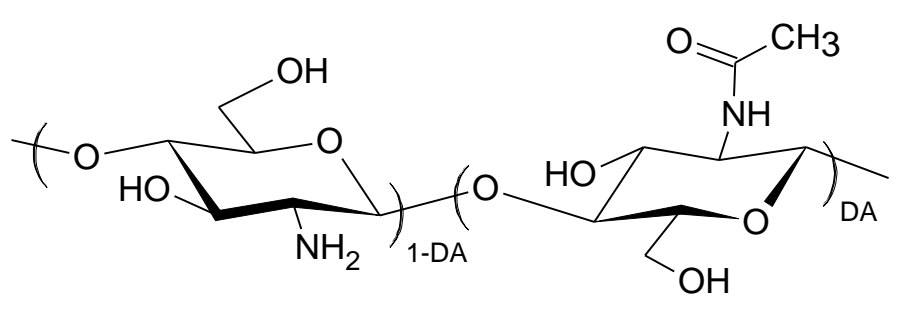

Chitosan

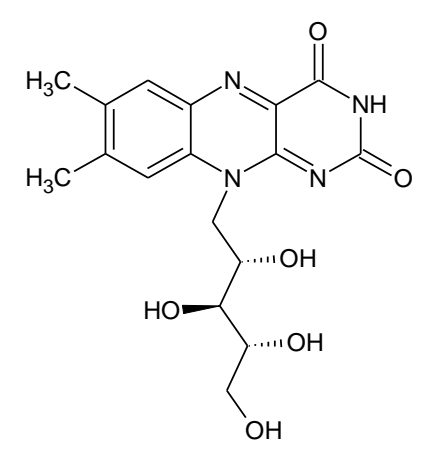

Riboflavin

Figure 1 : Chemical structures of chitosan and riboflavin. 


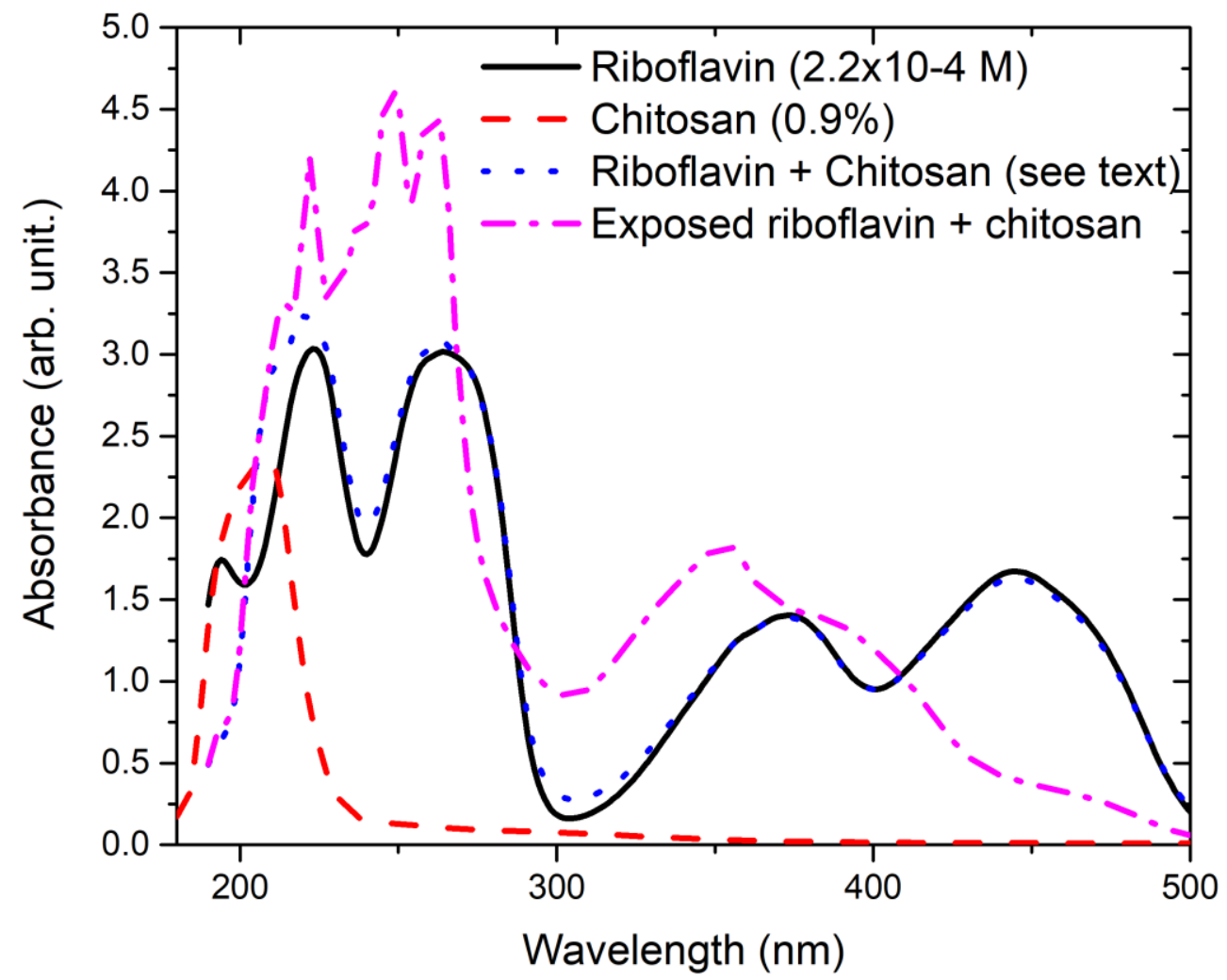

Figure 2: Absorbance spectra of the UV-exposed and non-exposed mixture of the $2.2 \times 10^{-4}$ mol. $L^{-1}$ riboflavin and $0.9 \%$ chitosan solutions compared to non-exposed solutions of $2.2 \times 10^{-4}$ mol.L $L^{-1}$ riboflavin and $0.9 \%$ chitosan solution. 


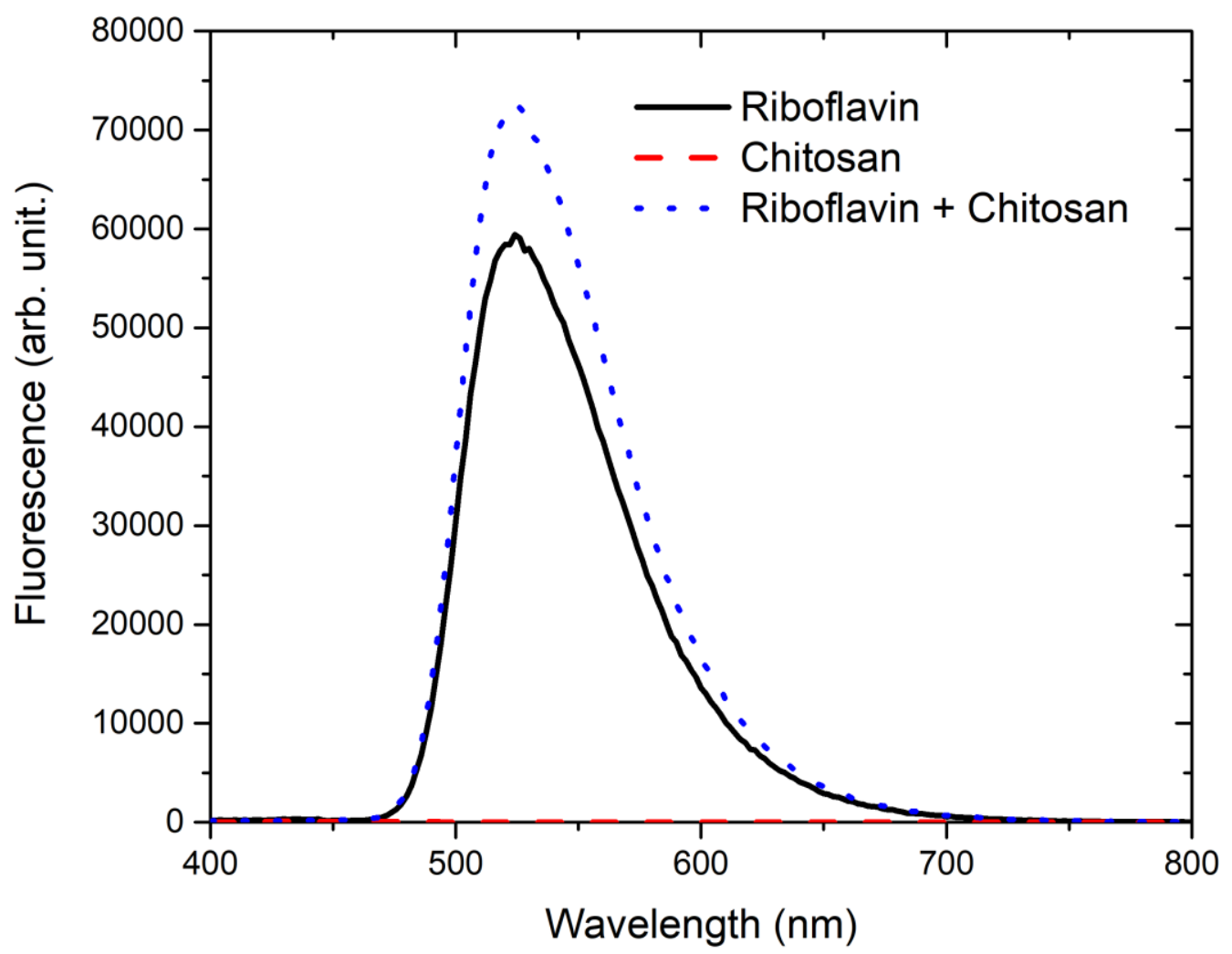

Figure 3: One Photon Excitation Fluorescence (OPEF) spectra of pure riboflavin, pure chitosan and riboflavinchitosan mixture solutions excited at $377 \mathrm{~nm}$. 


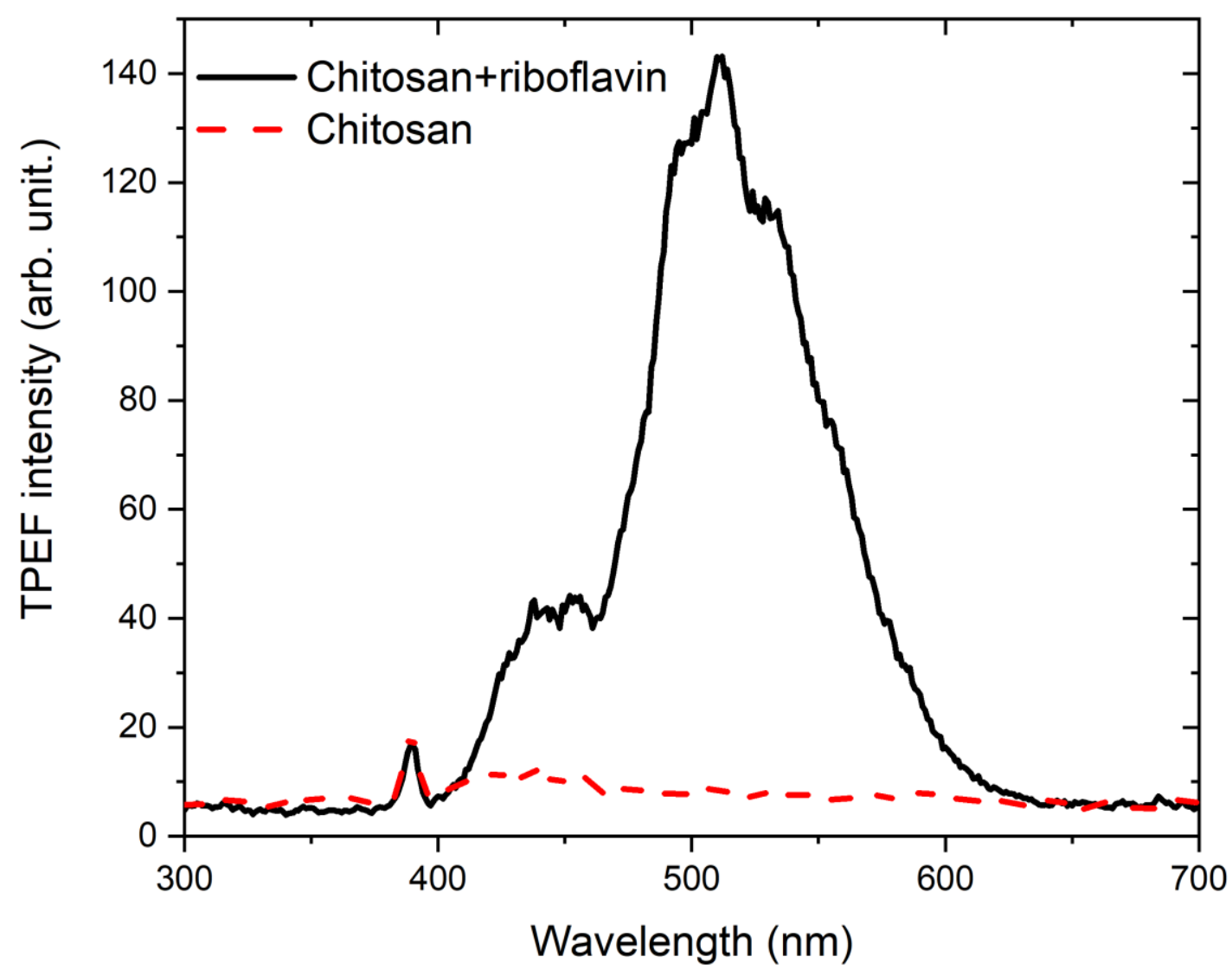

Figure 4: Reflectance of the silica substrate and the silica substrate coated with chitosan and a mixed riboflavinchitosan film.

Figure 5 : TPEF spectra for a pure chitosan film and a mixed riboflavin-chitosan film with a $780 \mathrm{~nm}$ wavelength excitation. An SHG peak is observed in both samples at $390 \mathrm{~nm}$. 

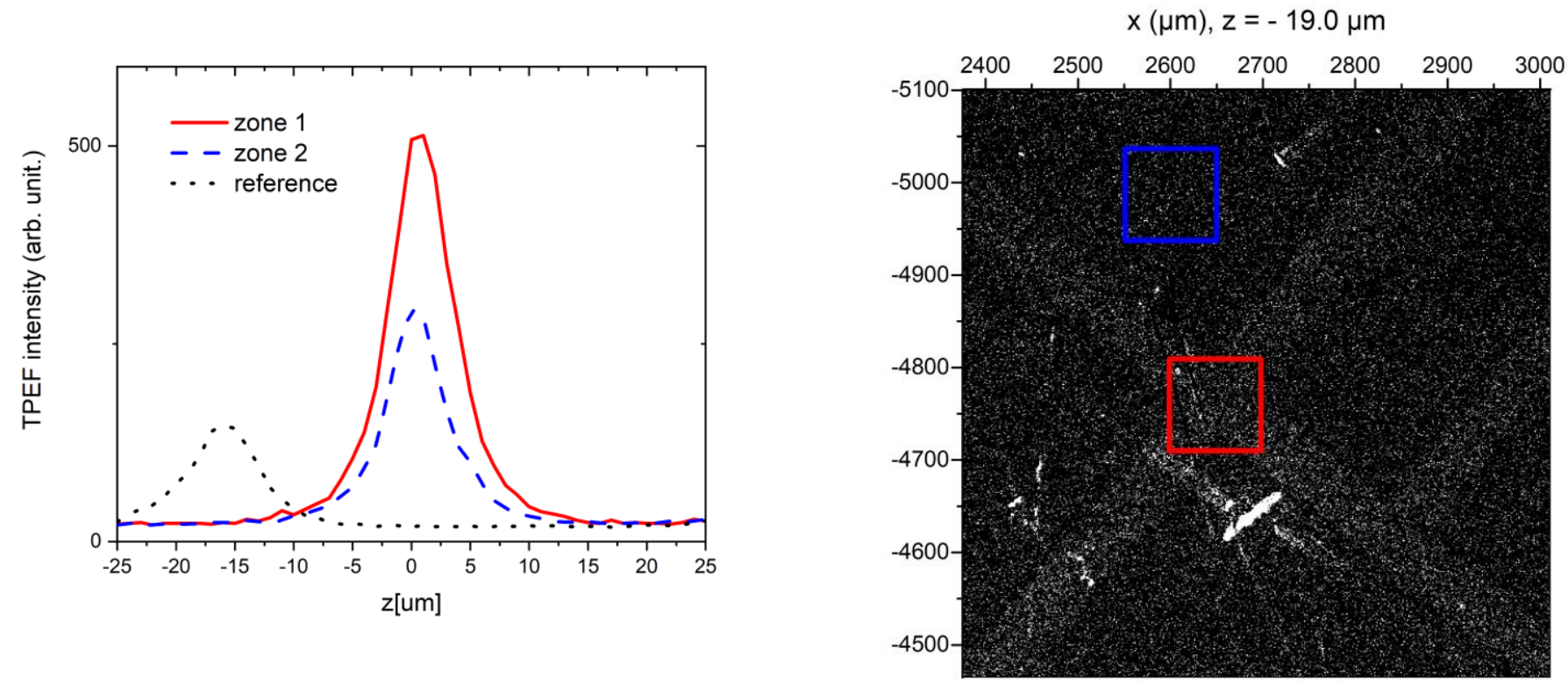

Figure 6: (a:left) TPEF signal integrated over the 410 - $650 \mathrm{~nm}$ spectral region as a function of the film depth for two different areas of the sample and the reference $\left(\mathrm{SiO}_{2}\right.$ substrate). (b:right) Image of the exposed sample as obtained at the depth giving the maximum TPEF intensity $(z=0 \mu m)$. 

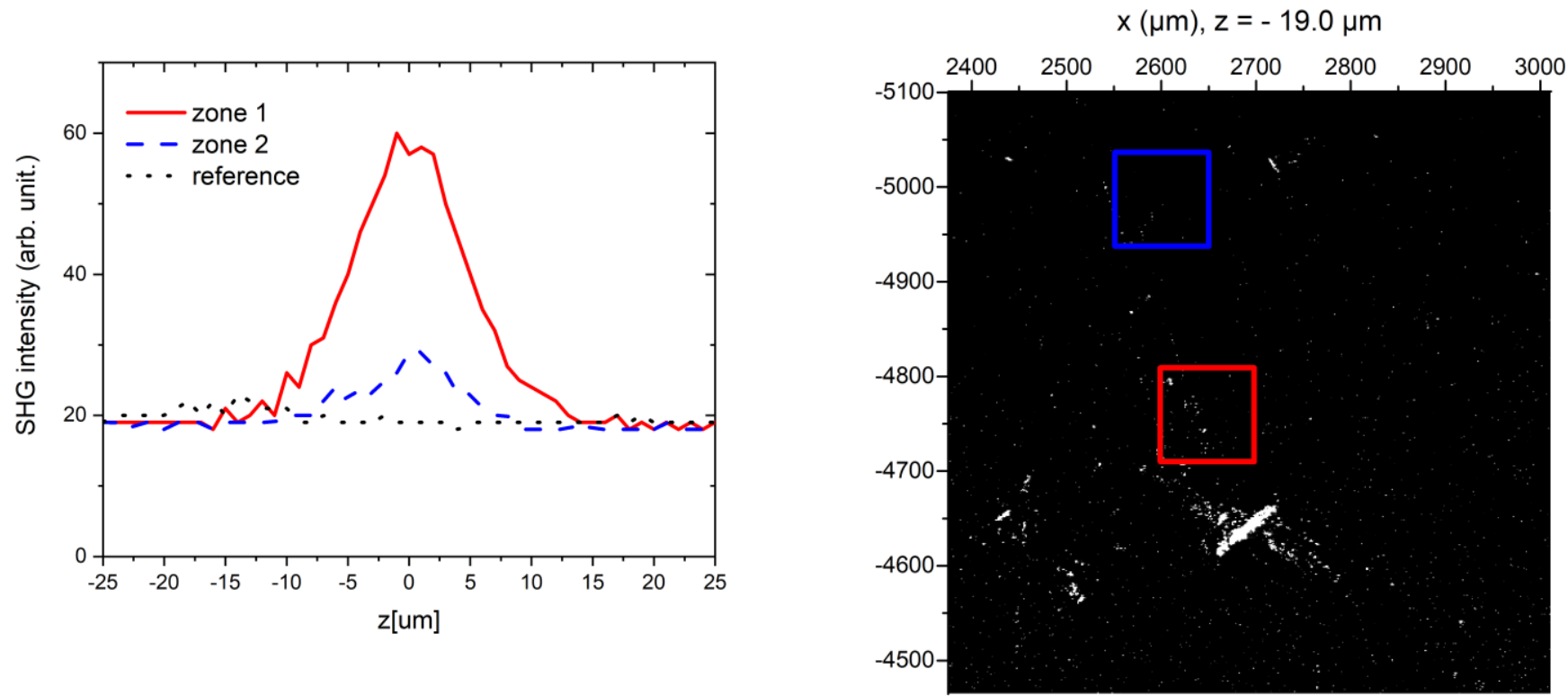

Figure 7: (a:left) SHS (second harmonic scattering) recorded at $390 \mathrm{~nm}$ as a function of the film depth for the same areas of the previous figure. (b:right) Image of the exposed sample obtained at the depth giving the maximum intensity of SHS ( $\mathrm{z}=0 \mu \mathrm{m})$.

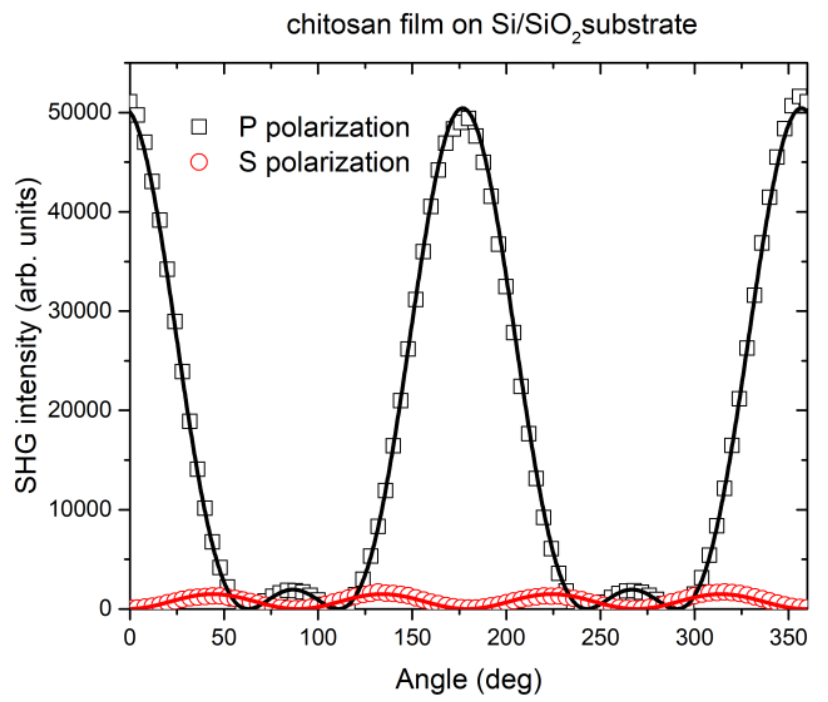

chitosan/riboflavin non exposed film on $\mathrm{Si}_{\mathrm{SiO}}$ substrate

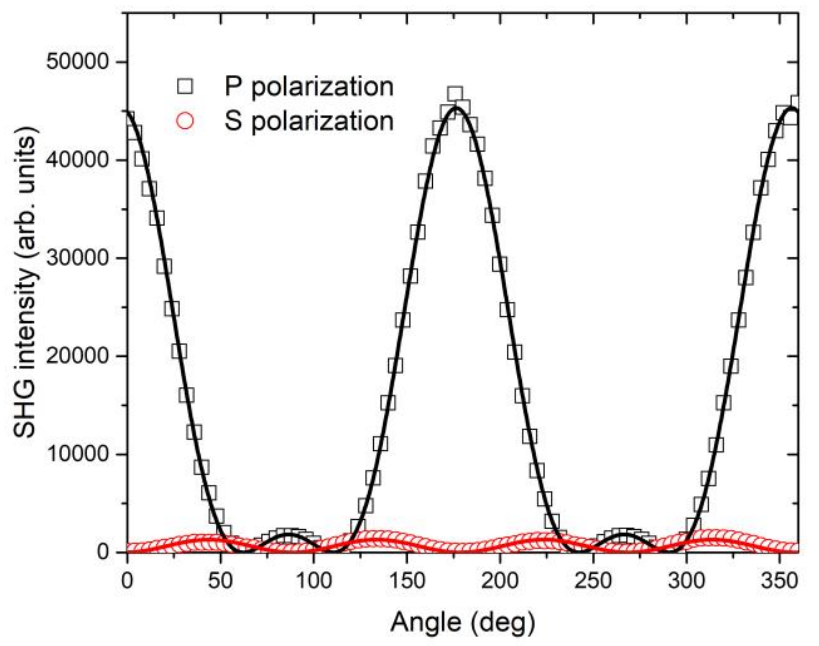

Figure 8: Surface SHG intensity as a function of the fundahthatal polarization angle for a chitosan film on Si/SiO ${ }_{2}$ substrate (a:left) and a non-exposed riboflavin-doped chitosan film on $\mathrm{Si}_{\mathrm{SiO}}$ substrate (b:right). The black squares and red circles curves correspond respectively to the $P$ - and $S$ - harmonic intensities ( $I_{P}$ and $I_{S}$ ) with their corresponding fits. 
Graphical Abstract

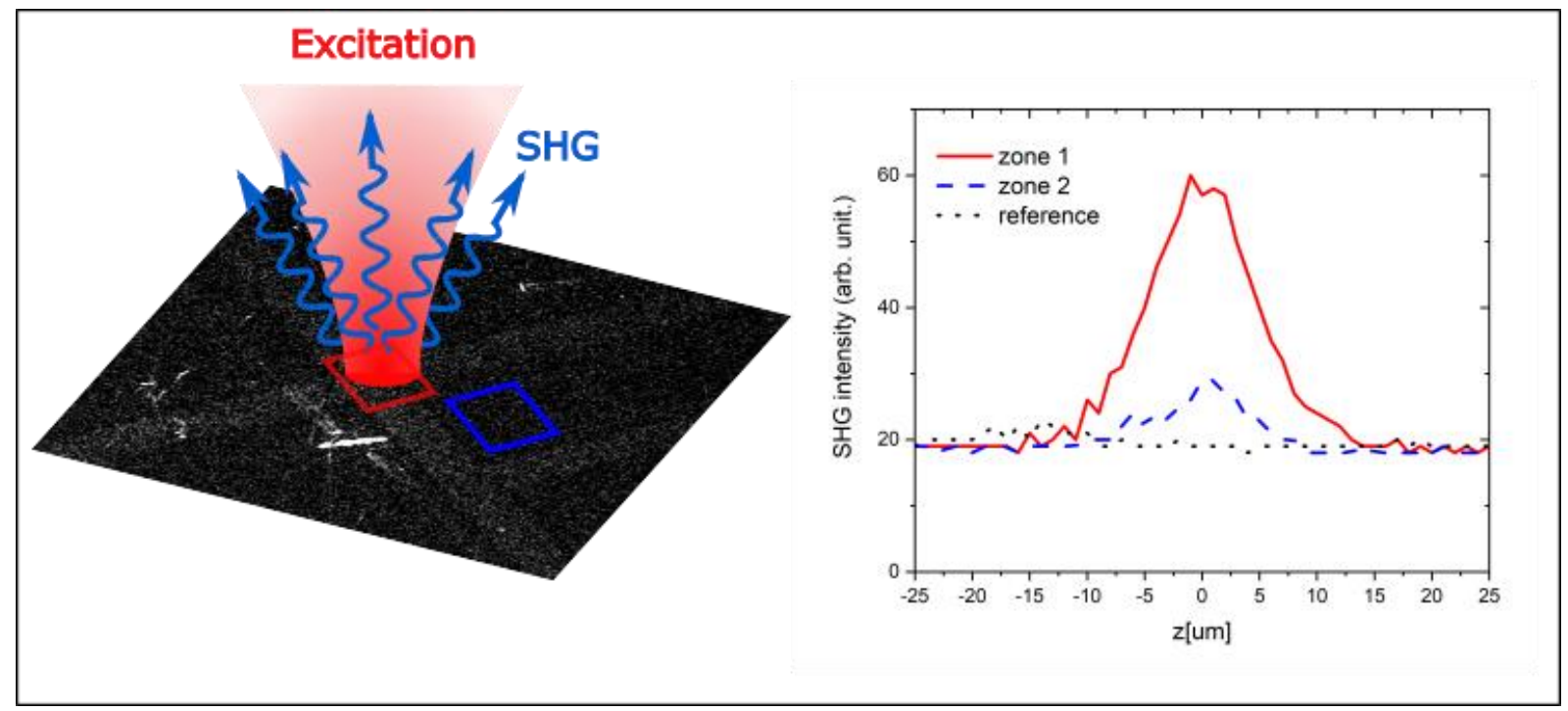

